Agrarholz als neue Perspektive für Landwirte

\title{
Potenziale und Rahmenbedingungen schnellwachsender Baumarten
}

\section{Agrarholz könnte in Zukunft einen wichtigen Beitrag zum Klimaschutz leisten. Damit er- öffnet sich für Landwirte eine weitere Option, zum Energiewirt und Rohstofflieferant zu wer- den. Gleichzeitig kann der Nutzungsdruck auf den Wald gemindert und der Konkurrenzdruck zu den Nahrungsmitteln entschärft werden. \\ Von Dieter Murach, Astrid Aretz und \\ Bernd Hirschl}

K imaschutz und die abnehmende Verfügbarkeit der fossilen Energieträger haben in den letzten Jahren einen Bioenergieboom ausgelöst. Dieser Boom hat zu einer gravierenden Zunahme der Nachfrage insbesondere nach Holz als dem bedeutendsten Biomasse-Rohstoff geführt. Die energetische Verwendung konkurriert dabei mit der stofflichen Nutzung, und steigende Preise können zu einer Belastungsprobe für eine nachhaltige Bereitstellung von Waldholz führen. Hier könnte die Landwirtschaft durch den Anbau von Agrarholz einen Teil der steigenden Nachfrage auf umwelt- und klimaschützende Weise befriedigen. Das vom Bundesministerium für Bildung und Forschung geförderte Forschungsprojekt Dendrom hat sich von 2004 bis 2008 in umfassender Weise mit dem Agrarholzanbau befasst, und dabei in einem Schwerpunkt Konzepte und Szenarien für Regionen in Brandenburg entwickelt, in dem bereits heute die Waldholzpotenziale nahezu ausgeschöpft sind (1).

Im Rahmen von Dendrom wurde erstmalig das sich abzeichnende Ungleichgewicht zwischen Angebot und Nachfrage von

Abbildung 1: Verwendungsmöglichkeiten von Holz

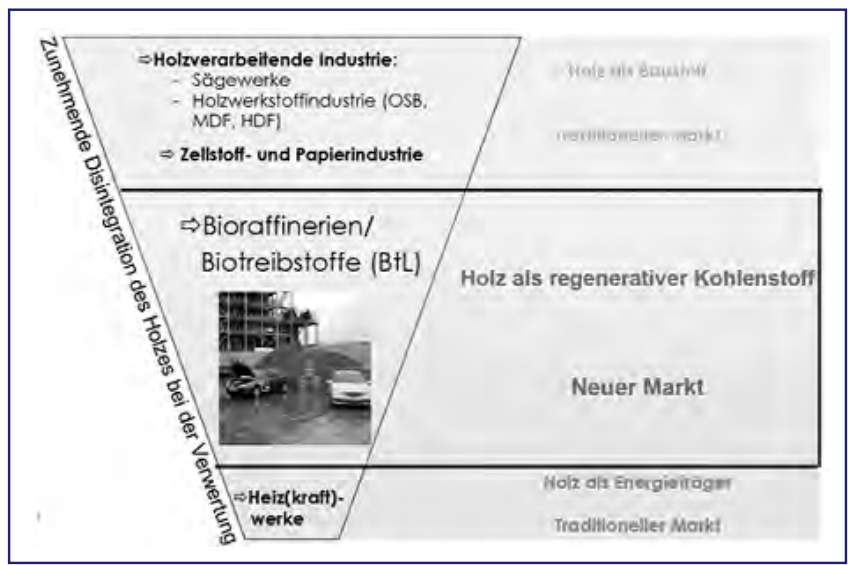

Quelle: Eigene Darstellung geringwertigen Holzsortimenten eingehender untersucht. Mittlerweile hat sich dieses Ungleichgewicht bestätigt (Mantau 2007). Die Nutzungskonkurrenz bei Holz für die energetische und stoffliche Verwendung ist bereits jetzt zu einer ernsten Herausforderung in vielen europäischen Ländern geworden. Zur Entlastung der europäischen Wälder formulierte die Sektion Holz der United Nations Economic Commission for Europe in einer Empfehlung an die Ministerkonferenz zum Schutz der Europäischen Wälder im November 2007 unter anderem den Anbau von schnellwachsenden Baumarten auf landwirtschaftlichen Flächen, also Agrarholz, zur Produktion holzartiger Biomasse beziehungsweise Dendromasse (Dengg 2007).

Der Nutzungsdruck wird jedoch auch durch neue Märkte für holzartige Biomasse, durch Bioraffinerien und die Kraftstoffindustrie der sogenannten zweiten Generation erhöht. Werke zur Produktion von Btl (Biomass-to-liquid)-Kraftstoffen im industriellen Maßstab, in Deutschland vor allem repräsentiert durch die Firma Choren, sind in der Planung. Ihr Bedarf an Rohstoffen wird, wenn sie realisiert werden, beträchtlich sein. Allein Choren kalkuliert für seine geplante industrielle BtL-Produktion mit über einer Millionen Tonne Holz pro Jahr (Deutmeyer 2007). Durch den thermo-chemischen Aufschluss der Biomasse mittels Pyrolyse und anderer Verfahren verschwimmt die Grenze zwischen stofflicher und energetischer Nutzung von Biomasse. Zukünftig werden Dendro- und Biomasse vor allem als Kohlenstoffquelle und damit als Ersatz in allen Bereichen eingesetzt, in denen fossile Rohstoffe genutzt werden.

\section{Besonderheiten des Agrarholzanbaus}

Die Bewirtschaftung von Wäldern in der Forstwirtschaft und von Agrarholz in der Landwirtschaft haben nicht viel miteinander gemein. Während der Förster seinen Wald in langen Produktionszeiträumen von etwa 100 bis 200 Jahren bewirtschaftet und bestrebt ist, einen möglichst hohen Wertholzanteil zu erzielen, arbeitet der Landwirt mit Rotationen von wenigen Jahren und dem Hauptziel, Dendromasse zu erzeugen. Er benötigt daher anders als der Förster Bäume mit hoher Massen- statt Wertleistung, einem raschen Jugendwachstum, einem guten Stockausschlagvermögen, da nicht nach jeder Ernte wieder neu gepflanzt werden soll, einer einfachen Vermehrbarkeit, hoher Anwuchssicherheit und geringen Anfälligkeit für Schäden. Aufgrund der geforderten Merkmale kommen in unseren Breiten vor allem die Gattungen der Pappel, Weide und Robinie in Betracht. Denkbar sind aber auch andere Baumarten wie zum Beispiel Aspe, Erle oder Birke. 


\section{Holzmarktsituation in Brandenburg}

Der Vergleich der aktuellen Verarbeitungskapazität der in Brandenburg ansässigen holzverarbeitenden Industrie mit dem nachhaltig verfügbaren Holzangebot aus Brandenburgs Wäldern zeigt, dass der Bedarf langfristig nicht befriedigt werden kann (Knur et al. 2008). Besonders deutlich wird die Diskrepanz zwischen Angebot und Nachfrage bei den geringwertigen Sortimenten Industrie- und Energieholz, obwohl hierbei nur die bereits bestehenden Kapazitäten und nicht die geplanten Großabnehmer wie zum Beispiel BtL-Werke berücksichtigt wurden. Die Lücke zwischen Angebot und Nachfrage nach Dendromasse in Brandenburg macht eine langfristig zunehmende Nachfrage nach Agrarholz wahrscheinlich. Die Frage ist nun, ob der Agrarholzanbau unter den standörtlichen Verhältnissen in Brandenburg auch ökonomisch konkurrenzfähig mit den annuellen Kulturen ist.

\section{Voraussetzungen für den Agrarholzanbau}

Ein wichtiger Faktor für das Wachstum von Pappel und Weide ist das Wasserangebot, das für die potenzielle Transpiration der Bäume zur Verfügung steht. Das Transpirationswasserangebot (TWA) wird bestimmt durch den Niederschlag, die Wasserspeicherfähigkeit des Bodens und die Grundwasserverfügbarkeit. Das Grundwasser spielt insbesondere im Tiefland von Brandenburg eine wichtige Rolle und kann hier das niedrige Wasserspeichervermögen der sandigen Böden und die geringen Niederschläge in Brandenburg vollständig kompensieren. Maximale Erträge lassen sich nur auf grundwasserbeeinflussten Standorten erzielen.

Ertragreiche Pappelklone zeigen insbesondere auf nicht grundwasserbeeinflussten Standorten selbst bei geringeren Pflanzenzahlen höhere Erträge als die Weidensorten, die bisher in die Untersuchungen eingingen. Optimale Wuchsleistungen werden bei den Weiden auf etwa 16 Tonnen atro pro Jahr und Hektar geschätzt, für die Pappeln auf etwa 20 Tonnen atro pro Jahr und Hektar. Die Ertragsschätzungen für Pappeln auf terrestrischen Standorten lassen auf gut wasserversorgten, aber nicht grundwasserbeeinflussten Standorten bei optimalen Bedingungen und einem Pflanzverband zwischen 5.000 und 10.000 Pflanzen pro Hektar Erträge von bis zu 15 Tonnen atro pro Jahr und Hektar erwarten. Als Richtwert für grundwasserferne schwachhumose, schwachlehmige Sandböden in Brandenburg mit einer Bodenzahl um 30 und durchschnittlichen Jahresniederschlägen von etwa 480 Millimeter kann für Pappeln bei einer Pflanzenzahl von ca. 12.000 Stück pro Hektar und Umtriebszeiten unter fünf Jahren von etwa 10 Tonnen atro pro Jahr und Hektar ausgegangen werden.

\section{Ökonomischer Vergleich mit annuellen Kulturen}

Der Anbau von Agrarholz weist im Vergleich mit annuellen landwirtschaftlichen Kulturen selbst unter umfassender Berücksichtigung vielfältiger gesetzlicher und naturschutzfachlicher Restriktionen bedeutende ökonomische Potenziale auf. Beschränkt man das Agrarholzpotenzial aus Gründen einer Risikominimierung für die landwirtschaftlichen Betriebe auf grundwasserbeeinflusste Standorte, wo Agrarholz seine höchsten Erträge erzielen kann, dann ergibt sich als Minimum aller in Dendrom entwickelten Szenarien im Jahr 2030 eine Anbaufläche von etwa 30 Prozent der aktuellen landwirtschaftlichen Fläche mit einer Gesamtagrarholzmenge von etwa fünf Millionen Tonnen atro. Hierbei sind neben den verfügbaren Techniken auch konkurrierende Nutzungen auf Grundlage eines ökonomischen Vergleichs mit annuellen Kulturen auf Gemeindebasis enthalten. Ebenso wurden strukturelle, ökologische und weitere nichttechnische Beschränkungen wie beispielsweise der Abzug von Flächen innerhalb von Naturschutzgebieten, Abzug der Pufferzone von drei Metern um alle Oberflächengewässer sowie der Abzug von Ökolandbauflächen berücksichtigt.

Dieses hohe Flächenpotenzial in Brandenburg ist zunächst überraschend, da die Abhängigkeit des Wachstums von Pappeln und Weiden von der Wasserversorgung belegt und Brandenburg mit 450 bis 550 Millimeter das niederschlagärmste Bundesland in Deutschland ist. Die Erklärung liegt in den zwei Hauptregionen Brandenburgs mit grundsätzlich unter- $\rightarrow$

Abbildung 2: Ertragspotenziale von Weiden (linke Grafik) im Kurzumtrieb (3 Jahre) und Pappeln (rechte Grafik) im Kurzumtrieb 5 Jahre) bei verschiedenen Pflanzverbänden in Relation zum Transpirationswasserangebot

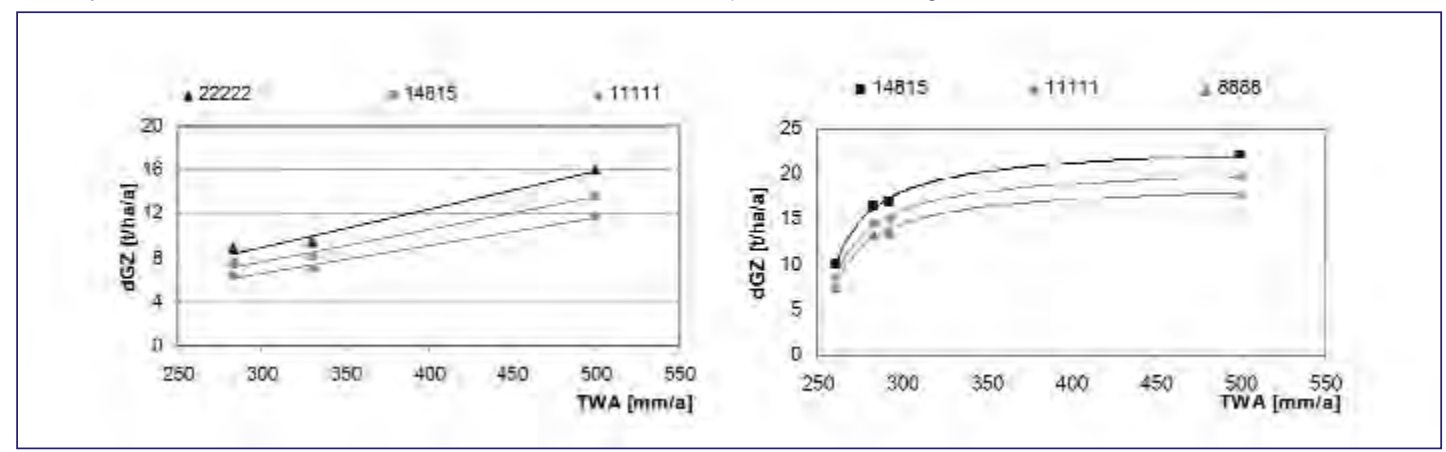

Quelle: Knur et al. 2008 
schiedlicher Wasserversorgung. Dies sind die Feuchtgebiete in den Niederungen und die grundwasserfernen Hochflächen (Eulenstein/Wenkel 2002). Ein Viertel der Fläche Brandenburgs und etwa die Hälfte der Ackerflächen sind grundwasserbeeinflusst. Vier Prozent der Landesfläche werden durch Gewässer eingenommen, die größer als ein Hektar sind. Damit zählt Brandenburg zu den gewässerreichsten Bundesländern. Die Niederungen werden durch etwa 25.000 Kilometer künstlicher Gräben entwässert (LUA 2004).

\section{Brandenburg prädestiniert für Agrarholz}

Trotz Regenarmut und geringer Wasserspeicherkapazität der Böden ist also im Tiefland genug Wasser für den Anbau von Agrarholz vorhanden. Gerade die Kombination der speziellen hydrologischen Verhältnisse und der Sandböden machen Brandenburg zu einem ausgesprochen prädestinierten Anbaugebiet für Agrarholz in Deutschland. Hinzu kommen weitere standörtliche Vorteile, die in der Betriebsgröße und den relativ geringen Preisen für das Agrarland liegen. Etwa 60 Prozent der Agrarfläche in Brandenburg, das sind etwa 600.000 Hektar, werden durch landwirtschaftliche Betriebe mit einer Größe von mehr als 1000 Hektar bewirtschaftet.

Trotz dieser regionalen Vorzüge des Agrarholzanbaus konnte sich dieses Landnutzungssystem in Brandenburg, wie auch bundesweit, bisher noch nicht etablieren. Die Gründe liegen in den allgemeinen, vorwiegend jedoch finanziellen Restriktionen bei der Implementierung von Agrarholz. Es werden spezielle Maschinen benötigt, die hohe Investitionen nach sich ziehen und eine Mindestanbaufläche verlangen, die in der Größenordnung von etwa 1000 Hektar liegt. Die Ernteerträge fallen im Unterschied zu den annuellen Kulturen nicht jedes Jahr an, was ein Liquiditätsproblem darstellen kann. Hinzu kommen Unsicherheiten bei den Ertragsprognosen.

\section{Restriktionen und aktuelle Entwicklungen}

Mittlerweile gibt es einzelne nicht landwirtschaftliche Investoren, die Agrarholz in großem Maßstab anbauen lassen wollen, wie zum Beispiel RWE Innogy, oder dies bereits tun, wie die Schellinger KG. Ob sich ohne Pilot- und Demonstrationsprojekte mit Unterstützung der öffentlichen Hand eine breitere Anwendung entwickeln wird, ist jedoch fraglich. Zudem wird dadurch möglicherweise die Chance vergeben, die ökologischen Vorteile, die der Anbau von Agrarholz bieten kann (Aretz/ Hirschl 2008), vollständig zu erschließen und gleichzeitig die möglichen visuellen und naturschutzbezogen Nachteile zu minimieren. Eine solche strategisch geplante Erschließung der Agrarholzpotenziale zeichnet sich gegenwärtig jedoch nicht ab. Eine besondere Dynamik, die möglicherweise stark durch kapitalkräftige und nicht unbedingt ökologisch motivierte Investoren geprägt sein wird, ist durch die Privatisierung der letzten Flächen der Bodenverwertungs- und -verwaltungs $\mathrm{GmbH}$ mit etwa 600.000 Hektar ab 2010 zu erwarten.

\section{Anmerkungen}

(1) Das Projekt Dendrom wurde gefördert im Rahmen des Förderschwerpunkts „Nachhaltige Waldwirtschaft“ im Rahmenprogramm „Forschung für die Nachhaltigkeit (FONA) “. Mehr Informationen im Internet unter: http://www.dendrom.de

\section{Literatur}

Aretz, A. / Hirschl, B.: Holz von landwirtschaftlichen Flächen. Eine Option zur Angebotserhöhung und ihre ökologischen Wirkungen. In: Ökologisches Wirtschaften 1/2008, S. 19-21.

Dengg, J.: Chaiman's summary. Joint UNECE Timber Committee and FAO European Forestry Commission Policy Forum on "Opportunities and Impacts of Bioenergy Policies and Targets on the Forest and Other Sectors" in Geneva, 10 Oct. 2007. Internet: http://www.unece.org.

Deutmeyer, M.: Strategies for Supply of Raw Material in the Energy Industry CHOREN's BtL Technology. UNECE Workshop „Mobilizing wood ressources". Geneva 11.-12.01.2007. Internet: http://www.unece.org.

Eulenstein, F. / Wenkel, K.-O.: Wasserrückhalt in Agrarlandschaften: Beitrag der Landnutzung an der Versickerung von Wasser zur Grundwasserneubildung am Beispiel Mittlerer Fläming. Landesamt für Verbraucherschutz und Landwirtschaft, 3. Brandenburger Beregnungstag, Güterfelde, 21.11.2002. S. 20-34. Internet: http://www.fachverband-feldberegnung.de.

Knur, L. / Murn, Y. / Murach, D. / Aretz, A. / Fiedler, P. / Bilke, G.: Szenarien und Handlungsempfehlungen für eine nachhaltige Dendromassebereitstellung in Brandenburg. In: Murach et al. (Hrsg.), Vorläufiger Endbericht des Verbundforschungsvorhabens DENDROM - Zukunftsrohstoff Dendromasse. Berlin 2008. Internet: http://www.dendrom.de.

LUA [Landesumweltamt Brandenburg]: Leitfaden zur Renaturierung von Feuchtgebieten. Studien und Tagungsberichte des Landesumweltamtes, Bd. 50. Potsdam 2004. Internet: http://www.mluv.brandenburg.de.

Mantau, U.: The legend of the woody biomass reserve in Europe. UNECE Workshop „Mobilizing wood ressources“, 11.-12.01.2007, Geneva. Internet: http://www.unece.org.

\section{【 AUTOREN + KONTAKT}

Dr. Dieter Murach ist Professor an der Fachhochschule Eberswalde im Fachbereich Wald und Umwelt.

Fachhochschule Eberswalde, Alfred-Moeller-Str. 1 16244 Eberswalde.

Dr. Astrid Aretz ist wissenschaftliche Mitarbeiterin, Dr. Bernd Hirschl ist Leiter des Forschungsfeldes Nachhaltige Energiewirtschaft und Klimaschutz am Institut für ökologische Wirtschaftsforschung (IÖW), Berlin.

IÖW, Potsdamer Str. 105, 10785 Berlin. Tel.: +49 308845940 ,

E-Mail: astrid.aretz@ioew.de, bernd.hirschl@ioew.de. Internet: www.ioew.de

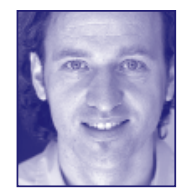


(c) 20I0 Authors; licensee IÖW and oekom verlag. This is an article distributed under the terms of the Creative Commons Attribution Non-Commercial No Derivates License (http://creativecommons.org/licenses/by-nc-nd/3.o/), which permits unrestricted use, distribution, and reproduction in any medium, provided the original work is properly cited. 